This is an author produced version of a paper published in New Media \& Society. This paper has been peer-reviewed but does not include the final publisher proof-corrections or journal pagination.

Citation for the published paper:

Klinger, Ulrike; Svensson, Jakob. (2018). The End of Media Logics? On Algorithms and Agency. New Media \& Society, vol. 2018, issue 12, p. null

URL: https://doi.org/10.1177/1461444818779750

Publisher: Sage

This document has been downloaded from MUEP (https://muep.mah.se) / DIVA (https://mau.diva-portal.org). 


\title{
The End of Media Logics? On Algorithms and Agency
}

\author{
Abstract: \\ We argue that the design of algorithms is an outcome rather than a replacement of \\ media logics, and ultimately we advance this argument by connecting human agency to \\ media logics. This theoretical contribution builds on the notion that technology, \\ particularly algorithms are non-neutral, arguing for a stronger focus on the agency that \\ goes into designing and programming them. We reflect on the limits of algorithmic \\ agency and lay out the role of algorithms and agency for the dimensions and elements \\ of network media logic. The article concludes with addressing questions of power, \\ discussing algorithmic agency from both meso and macro perspectives.
}

Keywords: algorithms, agency, media logic, digital communication, neutrality

\section{Introduction}

Algorithms are on the agenda today. Examples are abundant. How Facebook manually controls the algorithms by tweaking them (Tufekci, 2015), the debate whether Amazon is homophobic (Striphas, 2015), whether Google is racist (Allen, 2016) and the scandal 
over Microsoft's chat program Tay that quickly turned to obscene and inflammatory language after having interacted with Twitter users (Neff and Nagy, 2016). Studies have also found gender biases as a consequence of image search algorithms (Kay, Matuszek and Munson, 2015) and that black people are not recognized as humans in facerecognition algorithms (Sandvig et al., 2016).

Within the field of social media and communication, thinking has also taken an "algorithmic turn" (Napoli, 2014). Scholars argue that algorithms are becoming increasingly important - to the degree that they start to replace many things, from production to consumption of media (Napoli, 2014), from editors (DeVito, 2016) to journalists (Anderson, 2013; Van Dalen, 2012), and might even influence election results (Tufekci, 2015, p. 204). Steiner's 2012 book, subtitled "how algorithms came to rule the world," is a case in point: In it, he argues that algorithms control financial markets, the music that reaches our ears, and even how we choose a partner. Indeed, algorithms are more and more responsible for selecting the information that reaches us (Gillespie, 2014), which has consequences for the shaping of our social and economic life (Kitchin, 2017). Studies have revealed, for example, how the algorithmically generated news feeds containing our acquaintances' actions and opinions influence the issues on our agenda and how these issues are framed (Just and Latzer, 2016, p. 10), which in turn influences our decisions and preferences (Tufekci, 2015). Facebook and Google are replacing traditional media channels as information intermediaries (Bozdag, 2013) to the point that Facebook now is the number one source of news about government and politics for a majority of the so-called "millennials" (Diakopoulus, 2016). Connected to this is the notion that data could speak for itself, with algorithms 
identifying patterns and generating knowledge without the bias of scholars and their predetermined research questions (Just and Latzer, 2016). Anderson (2008) famously even argued that we might not need theory anymore.

In this article, we connect this discussion with another debate in the field: Which logics prevail on social media platforms (see van Dijck and Poell, 2013; xxxxx 2015, 2016)? As we will discuss in greater detail below, the theory of media logics is based on the observation and empirical evidence that there are some inherent rules of the game in how media works. The media logics theory addresses questions around how content is produced, how news are selected, how information are distributed and circulate and how people use media, whether it is new or traditional media in hybrid media systems (so-called network media logic). Now algorithms entered the scene, making (more or less) automated decisions in all the areas that have so far been addressed within the theory of media logics. Thus, it is time to raise the question of media logics and algorithms. We ask: Is it at all possible to make an argument about network media logic if one assumes that increasingly autonomous algorithms shape the functions and experiences of social media platforms? Is the purpose for the logic of social media platforms to be reduced to the relevant algorithms and how they operate? We will answer No to this question, for the simple reason that algorithms are not neutral. As we will develop below, they are programmed and engineered by human actors and thus rather an outcome of media logics. Understanding algorithms as socio-material processes we will differentiate different steps in these processes, and propose a way to think about agency (human as well as non-human) in these different steps. We end the article with a discussion of how human agency is deeply ingrained in all elements of 
network media logic. First we attend to algorithms and how they can be understood, before we turn to network media logic and the question of actors and agency in this techno-social setting.

\section{On Algorithms and Media Logics}

\subsection{Algorithms}

It is not easy to define algorithms. Reasons for this are that the notion is sloppily used (Sandvig et al., 2016, p. 4975), the notion is expanding (Gurevich, 2012) and has developed into somewhat of a modern myth (Barocas, Hood and Ziewitz, 2013). Etymologically the word algorithm comes from the Greek word of number "arithmos" and the Arabic word for calculation "al-jabr" (from which algebra stems, see Striphas, 2015). Kowalski made an early attempt in 1979 by discussing algorithms as problemsolving technologies that consist of a logic component specifying the knowledge (data) to be used in the problem solving and a control component, a calculation, determining how the knowledge should be used to solve the problem. They comprise of both output as well as input (Seaver, 2013; Kitchin, 2017), code that defines how their calculations should be executed and what data should be included in the calculations. Hence, algorithms should be understood as material as well as social processes as their calculations may be based on direct articulations from the programmer, on data they draw their calculations from, or data generated from calculations in previous steps. Algorithmic processes consists of many steps of which we crudely can discern a) input, the designing/programming (often based around problems that need to be solved), 
which b) leads to the formulation of one (or several) calculations which operate in a big-data context, calculations that then c) result in some kind of outcome (output).

Algorithmic calculations operate in big data contexts. The amount of information that we create and leave behind when reading, liking and sharing online has been multiplying, not the least because of computers and their storing and tracking capabilities. But more than its size, big data characterizes data that can be searched, aggregated and triangulated with other sets of data (Shorey and Howard, 2016, p. 5033). Terms such as "trace data" (Jungherr et al., 2016) and "thick data" (Langlois and Elmer, 2013) have also been used. It is in this context that algorithms make quick calculations, sort, filter, rank, profile users and weigh data for different purposes (Bozdag, 2013).

In popular science there is often an assumption that algorithms perform their calculations in a non-biased way, that data can speak for itself with algorithms identifying patterns and generating knowledge without the bias of humans: that algorithms calculate fairly and accurately, free from subjectivity, error, and attempted influence (Gillespie, 2014; Kitchin, 2017). It has also been argued that algorithmic governance is more evidence-based and data-driven than traditional governance (see Just and Latzer, 2016). Boyd and Crawford (2012) have addressed this notion as a "mythology" that surrounds applications of "big" or trace data: "the widespread belief that large data sets offer a higher form of intelligence and knowledge that can generate insights that were previously impossible, with the aura of truth, objectivity, and accuracy" (p. 663). Algorithmic calculations indeed deliver results with a kind of detachment, objectivity and certainty (Ananny, 2016, p. 98). However, there is near 
consensus among the critical scholarly literature about the non-neutrality of technology in general and algorithms in particular (e.g. Gillespie and Seaver, 2016 for a comprehensive overview).

\subsection{Network media logic}

Before we attend to the question whether algorithms replace media logics, we will briefly summarize the argument for media logics and our account of network media logic. In his 2014 update of the original concept, Altheide defines media logic as:

a form of communication and the process through which media transmit and communicate information. A basic principle is that events, actions, and actors' performances reflect information technologies, specific media and formats that govern communication. (...) Media logic refers to the assumptions and processes for constructing messages within a particular medium. (Altheide, 2014, p. 22)

On this basis, we understand media logics as rules of the game of particular media, meaning the specific norms, rules, and processes that drive how content is produced, information distributed, and various media are used. We have previously outlined how media logics differ (although they do overlap) on social media compared with traditional mass media. For analytical purposes, we differentiated between media production, distribution, and usage (see $\operatorname{xxx} \operatorname{xxxx} 2015$ for the full account). It is obvious that algorithms are at play in all these areas. Concerning the production of media content, it has been well established that information in mass media is selected based on news values, while the logics behind posting on social media platforms are instead guided by authors' selection of information that is of personal interest to them. 
Algorithms that influence what information reaches us online are thus important for the production of media content online.

Whereas mass media targets, to a large extent, geographically defined communities (audiences), social media platforms are more bound to communities of peers and likeminded individuals. This means that information on social media platforms may reach a large number of self-selected like-minded others but rather not a general public. The distribution of information on social media platforms thus largely follows, ideally, the logics of virality, a network-enhanced word of mouth (Nahon and Hemsley, 2013). If information posted on social media platforms does not have the connective quality that encourages users to pass it on to like-minded others, it will not reach beyond a very limited circle (Bennett and Segerberg, 2012, p. 8). Algorithms play a role in privileging the popular and establishing connections between like-minded. While professionals working for traditional mass media usually know that the information they provide will reach a certain number of subscribers and viewers, the same information on social media platforms first has to be found and subsequently distributed by and among networks of like-minded others. Distribution on social media platforms greatly depends on like-minded and popular online intermediaries who serve as catalysts rather than as professional gatekeepers. As Bell (2016) has argued, social media has also had a disrupting effect on traditional mass media to such an extent that news publishers have lost control over distribution. Distribution has moved away to social media and platform companies that publishers could not have built even had they wanted to. It is filtered through algorithms and platforms which are opaque and unpredictable (see also Bozdag, 
2013 and Diakopuolus, 2016). Here we also glimpse the idea of algorithms as black boxes that are decisively changing the rules of the game of mass media.

With regard to media use, today we have to navigate within a landscape marked by an increasing abundance of information. We continuously need to make choices with regard to what among all this information is relevant for us, which is why content sharing and suggestions from popular and like-minded others within our social networks have become influential. Our networks inform us about the variety of choices before us, but above all how peers and like-minded others have acted and done in similar situations with similar choices (van Dijck, 2013). Hence, when connecting to likeminded and peers on social media platforms, users indirectly tailor the information that will reach them. In other words, to an increasing degree (compared to before social media), we construct and organize our social realities through our online social networks. News feeds of selected peers' likes, dislikes, and behaviors enable users to anticipate their future needs and wants based on an aggregate of their own and others' choices in the past. Such news feeds on social media are largely algorithmically steered.

Lately we have explored the concept in terms of how ideals, commercial imperatives, and technological affordances differ on mass media platforms compared with those on social media platforms (see $\operatorname{xxxxxx} 2016$ for the full account). Professional ideals in news production follow news values and vice versa (Strömbäck and Esser, 2014, p. 382). Ideals differ on social media platforms that rather follow values of produsage, connectivity, virality, and reflexive sharing of information among peers and likeminded individuals. Mass media is also mostly set in commercial landscapes that 
influence the ways in which content is produced, distributed, and used. They have to compete for audience attention and subscriptions, as well as advertising revenue, while at the same time keeping down the cost of production and dissemination in order to make money. In turn, the commercial imperatives on social media platforms are based on personal revelations and connectivity that are surveilled, mined, and used for targeted advertisements, not seldom with the help of algorithms. Technological affordances also shape the processes of producing, finding, and reproducing content. In the words of Hjarvard (2013), each media technology has characteristics that both enable and restrict media in their production, processing, and presentation of content. Social media platforms afford interactivity and push for constant updating in fragmented publics.

This conception of media logic is concerned with the meso level of organizations or even the macro level of society and the public sphere, rather than based on the micro level of individuals. Professional ideas, commercial imperatives and technological affordances refer to society, the economy and even our public spheres. So far however, algorithms have primarily been discussed on the micro level in terms of their their effects on individual users etc. By connecting algorithms to media logics we move the discussion up to meso and macro levels of analysis - in theory and empirical studies.

\subsection{Are algorithms replacing Network Media logics?}

It is obvious that algorithms impact different stages and elements of network media logic. However, does this mean that algorithms are replacing network media logic? Can network media logic be reduced to the defined steps of the algorithms at play in the 
particular social media platforms that populate contemporary communication landscapes? If this were the case, why still talk about media logics?

Our argument is that viewing algorithms as replacements for media logics would be both ideological and misleading. Understanding algorithms as non-human actors making rational decisions on behalf of humans but without human bias, is reminiscent of early critical thinking about science and technology as ideology (e.g. Habermas 1968):

For the concept of technical reason is itself perhaps ideology. Not merely its application, but technique itself is domination - over nature and over men: methodical, clairvoyant domination. The aims and interests of domination are not 'additional' or dictated to technique from above - they enter into the construction of the technical apparatus itself. For technique is a social and historical project: into it is projected what a society and its ruling interests decide to make of man and things. (Marcuse 1965, p. 16)

A few well-known statements from the innovators of now omnipresent digital media, Google and Facebook, illustrate this point that there is indeed ideology (understood here as specific ideas about the world and the human condition) inscribed into the code of these media entities: Facebook CEO Mark Zuckerberg's "Having two identities for yourself is an example of a lack of integrity" (Helft, 2011), or then-CEO of Google, Eric Schmidt's infamous "If you have something that you don't want anyone to know, maybe you shouldn't be doing it in the first place" (Huffington Post, 2010). Social media business models thrive on truthful self-disclosures and the massive and multilayered forms of surveillance (commercial surveillance, state surveillance, complicit surveillance, social surveillance, interveillance, etc.) these platforms afford (see, van 
Dijck, 2013). Hence, to argue for the rational and neutral workings of technology when pushing for self-disclosures online is indeed ideological.

Arguing that algorithms are replacing media logics would also point to a misunderstanding of algorithms (Gillespie, 2016; Kowalski, 1979), ignoring the input step (the programing and design) of algorithmic calculations. As Kitchin (2017) has pointed out, algorithms do not act in rational and orderly processes: They are not small black boxes performing single operations but "uncertain, provisional and messy fragile accomplishments" (p. 10) that were "teased into being: edited, revised, deleted, restarted, shared with others, passing through multiple iterations" (p. 10) over time and space. Problem-solving based on algorithmic calculations requires programmers and software engineers' input of relevant criteria, a pre-selection of possible outcomes, and clear instructions (see the long list of necessary human inputs in Gillespie, 2016). Even self-learning algorithms are not independent of human actors: They can only learn what and how they were programmed to learn. Or to put in into the terminology of AI (artificial intelligence) research: Today we can only achieve narrow AI, neither strong AI (which includes abstraction and contextual adaption) nor super-intelligence (Hindi, 2017).

Being designed and programmed by humans working in and for organizations, algorithms embody social values and business models. They are encoded with human and institutional intentions (that may or may not be fulfilled). As Lewis and Westlund (2015) argue: Technology is "inscribed and instructed by humans, socially constructed to suit journalistic, commercial and technological purposes within news organizations" 
(p. 24). If algorithms are shaped by human action and programmers' intervention, it is obvious that they do not/cannot replace media logics. Rather, they would be outcomes/manifestations of media logics, i.e. of the norms and processes of media production, distribution and usage, as well as how programmers and users perceive these norms and processes that go into the design/programming process. As Seaver put it:

\begin{abstract}
Algorithmic systems are not standalone little boxes, but massive, networked ones with hundreds of hands reaching into them, tweaking and tuning, swapping out parts and experiencing with new arrangements (...) we need to examine the logic that guides the hands. (Seaver, 2013, p.10, our emphasis)
\end{abstract}

Once we accept that algorithms are not neutral and do not replace network media logic, that programmers and software engineers in organizations are pivotal for algorithmic calculations and their outcomes, we must explore their agency. The above discussion underlines the importance of theorizing agency in the intersection of algorithms and media logics. Also, media logics are shaped by agency since rules, norms and processes both influence and are influenced by the involved actors, their actions, intentions, and perceptions. In the following we will therefore propose a way to think about the agency of the human and institutional actors behind algorithms, the agency of algorithms themselves in their different steps, and how agency relates to network media logic.

\title{
3. Agency
}

Concerning agency, we can identify two rather extreme poles of definition: one that limits agency to humans and one that not only acknowledges non-human actors but sees them on par with human agency. The first approach has a long tradition in the social sciences and may be best reflected in Arendt's (1958) notion that "(a)ction without a 
name, a 'who' attached to it, is meaningless" (p. 181). The latter one has most prominently been attributed to the proponents of actor-network theory (ANT), in which actors can have "human, nonhuman, unhuman, inhuman characteristics" (Latour, 1996, p. 7) because of ANT's "complete indifference for providing a model of human competence" (p.7).

Concerning algorithms, their agency is shaped both by humans in organizations and technology. In the input step of algorithmic calculations the agency is obviously human and would be affected by programmers and software engineers background, hacker culture and the contexts (commercial as well as organizational) in which the problems that the algorithms is designed to solve are formulated. The agency of algorithmic calculations once they are programmed, is less human and more shaped by the big/ thick /trace data that they filter, sort, weigh, rank and reassemble into some sort of outcome. The outcomes are based on the agency in the previous two steps, by the data they include in their calculations and how they are designed to calculate. It is here that algorithms are most visible for users, especially when they fail (Ananny, 2016, p. 989). One well-known case is Google's image search algorithm resulting in a multitude of (for sale) stock photography when searching for "three white teenagers". A search for three black teenagers, however, resulted in mugshots (see Allen, 2016). Google was thus accused for being racist.

While we cannot recap the long debate about agency in the social sciences, our argument builds upon Emirbayer and Mische (1998). They define human agency as a "temporally embedded process" (p. 962) that consists of three elements, each 
connecting agency to the past, future, and present. In their conception, human agency is:

the temporally constructed engagement by actors of different structural environments - the temporal-relational context of action - which, through the interplay of habit, imagination, and judgement, both reproduces and transforms those structures in interactive response to the problems posed by changing historical situations. (p. 970)

Following an impressive review of literature, Emirbayer and Mische (1998) identify three "constitutive elements" of human agency: iteration (linking action to the past), projectivity (linking action to the future), and practical evaluation (linking action to the present). In other words, agency depends on routinized practices (past - iteration), goal seeking and purposive activities (future - projectivity), and deliberation and judgment over the present situation.

The iterational element of agency is defined as "the selective reactivation of past patterns of thought and action (...) helping us to sustain identities, interactions and institutions over time" (Emirbayer and Mische, 1998, p. 971). This captures the first step in algorithmic calculations, how they are designed to select, recognize types, locate categories, sort and rank big/thick/trace data from the past. The projective element of agency refers to the "imaginative generation of actors of possible future trajectories of actions in which received structures of thought and action may be creatively reconfigured in relation to actors' hopes, fears, and desires for the future" (p. 971). Given that social media algorithms (to our knowledge) are designed to identify future purchasing possibilities for advertisers by algorithmically mining users' past behavior, we can argue that outcomes of algorithmic calculations are deeply connected to this projective element of agency. In other words, algorithms base their calculations on the 
past (available from big/trace/thick data) in order to project the future, most often in the service of advertisers, serving the business models of the social media companies for which algorithms are designed. In this way, the projective elements of social media's algorithmic calculations are both based on users' past behaviors and friend lists, as well as the commercial purposes of the companies to which they belong.

While algorithms account for some projectivity, they cannot move beyond themselves and are not able to change their relationship to their design; they cannot replace the human and institutional actors at the input stage. Even self-learning algorithms cannot (yet) learn and transform themselves beyond the outcomes they were designed to result in (Hindi, 2017). One example is Google's AlphaGO algorithm, which won the Chinese strategy game "Go" against world champion Lee Sedol in March 2016. The point is not that Google's developers programmed a self-learning algorithm with one superior Gostrategy, but that they programmed an algorithm that can teach itself how to play Go. Thus, the AlphaGo algorithm is in some ways able to anticipate and to project, although it remains unable to move beyond itself, or beyond the Go structure or Go orientation the designers had intended - for example, by teaching itself how to play chess instead of Go.

Future alternatives are not neatly presented, and "the future is not an open book" (Emirbayer and Mische, 1998, p. 989). It is primarily in the messy situations and contexts of the present that outcomes of algorithmic calculations can lead to unintended and undesirable consequences. This is true of human agency as well. But human agency, to a larger extent, builds on problematization (recognizing ambiguous 
situations), characterization (typifications), deliberation (of options), decision, and execution. It refers to "the capacity to make practical and normative judgements among alternative possible trajectories of action, in response to the emerging demands, dilemmas and ambiguities of presently evolving situations" (Emirbayer and Mische, 1998, p. 971, our emphasis). Human agency develops within the contexts and situations in which humans find themselves. But these are always changing, and we cannot always anticipate them. And what cannot be anticipated cannot be accounted for in the design/ engineering of algorithms. Algorithms lack a "reflective intelligence" (pp. 967-968) or a "deliberative attitude" (Mead, referred to on p. 969). Jansen (2016) argued recently that the idea of non-human agency goes "hand-in-hand with a very flat theory of agency, defining agents as those things and persons that make a difference in a situation" (p. 255) because it ignored the concept of reflexivity. He proposes "a purely formal notion of actors (...), which conceptualizes actors as producers of difference, thus connecting agency with reflexivity without binding reflexivity to a certain class of entities" (p. 256). The practical evaluative element of agency connects nicely to the reflexive, especially as Jansen (2016) defines it as "the act of perception, of noticing and pointing (...) the power of informing processes" (p. 259). This is where algorithms fail because they do not (yet) have this evaluative and reflexive element of agency.

This can further be linked to intentionality. Mitcham (2014) argues that intentionality implicates an "inner life that includes the ability to imagine objects and state of affairs, both past and prospective" (p.13) and that intentions are best described as "evaluative judgements" (p. 14). Algorithmic intentions are indeed connected to evaluating the past and prospecting the future, but cannot deal with the unexpected. This is where 
algorithms lack a reflexive capacity which implies that they cannot have any intentions of their own and can thus not be held accountable for their outcomes. Evaluation has to be ongoing and "temporally extended" (Mitcham, 2014, p. 14) to be effective, otherwise we will encounter algorithmic calculations that fail. Google's algorithm did not understand that its commercial projectivity could be considered racist and did not have the reflexive and evaluative capacities needed when the situation occurred. Google's algorithm was created by humans in organizations in a capitalist Western society, directed towards connecting sellers and buyers of stock photography, a majority of which are white. In the history of photography whiteness was early established as a norm - which has embedded photography in racist assumptions that 150 years later account for difficulties for non-white people to even be recognized by face-detection algorithms (see Sandvig et al., 2016). Still Google claimed that it was not them being racist but their algorithm (Allen, 2016). However, as Mictcham (2016: 15) argues, it is questionable if artifacts can have intentions as they have no inner life that they themselves can change. Therefore it is difficult to hold algorithms accountable, let alone blaming them for mistakes in their design. Human agency in contrast, develops as we confront emergent situations that have an impact on us. Hence, agency is not only teleologically oriented but situational, embedded in the handling of the contingencies of the present.

In conclusion, algorithms cannot independently change their "agentic orientations," and their actions are thus less complex than human actions: Human actors combine iteration, projectability, and practical evaluations because they "are always living simultaneously in the past, future and present, and adjusting the various temporalities of their empirical 
existence to one another in more or less imaginative or reflective ways" (Emirbayer and Mische, 1998, p. 1012). Algorithmic calculations are fueled by past experiences and thus, to some extent, may predict the future. They are also encoded with the commercial ends of the social media companies that developed them. But calculations that are left unmonitored cannot move beyond themselves or beyond the structures and the orientation their programmers intended. It is thus better to have algorithmic calculations monitored. In 2016, when Facebook fired its trending team which had been curating and babysitting the trending algorithm, this resulted in weird outcomes of their algorithmic calculations (to say the least, see Thielman, 2016).

\section{Network media logic and agency}

So far, we have concluded that algorithms are non-neutral, that algorithms do not replace media logic, and that they fail when it comes to the evaluative and reflexive part of agency. We have argued that algorithms are an outcome of media logics because they are incapable of moving beyond the structures and orientations intended by their programmers/ software engineers, who from the beginning are shaped by the perception of the logics of the media platforms they design for. In the final section of this article, we will argue that human and institutional agency shapes the logic on social media platforms and thus also algorithms.

Returning to previous discussions of how the media logics of mass media and social media platforms differ (but overlap), that media logics have three dimensions (content production, distribution of information, and media use), and that these dimensions consist of three elements (the underlying ideals, the commercial imperatives, and the 
technological affordances), we can systematically account for agency (in terms of iterations, projections, and evaluations) in these dimensions and elements.

\section{Insert here (Figure 1. Media Logic: Dimensions and Agency)}

\subsection{Ideals}

Starting with the underlying ideals of social media platforms, we have argued that values of constant updating, connectivity, and responsiveness shape the production, distribution, and usage of these platforms (xxxxxxx, 2016). Even though pushed to be updated, connect, and respond to others online, this is still to a large extent a result of human action. The iteration of past experiences, projections into future developments, and practical evaluations of current challenges shape the ideals that lie behind both traditional mass media and social media platforms. Ideals of how media content should be produced (e.g. professional ideals of news production, Esser, 2013), distributed or used are largely derived from past experiences - not least by glorifications of golden ages of journalism (e.g. Goodwin, 2014) that may have never existed (Big Think Editors, 2015).

\subsection{Commercial imperatives}

In practices of updating, connection, and response online, we leave a lot of trace data behind (Jungherr, Schoen, Posegga and Jürgens 2016), data that the business models of social media are built on (van Dijck, 2013). In other words, the commercial imperatives of social media are centered on humans actively and intentionally spending time on these communication platforms. By spending this time, they leave behind traces that are 
subsequently (and algorithmically) mined in order to surveil users with commercial intent, to target advertisements and so forth. Projections are encoded with commercial imperatives - projections about possible future buying activities by the user based on his/her past activities. The bond between commercial imperatives and agency runs deeper, however, because commercial imperatives are the outcome of past experiences, projections about how media markets will develop in the future, and practical evaluations of how to deal with current economic challenges. In hybrid media systems, this affects both traditional forms of (journalistic) mass media and the commercial imperatives behind social media platforms. Indeed, many problems of finding reliable business models in this new media landscape result from iterating past patterns in a new environment, such as trying to attract a mass audience where there is none (see e.g. Altheide's 2014 argument on the disappearing audience) or evaluating the success of a story by simple click rates, instead of becoming more responsive to reader's preferences by implementing more refined measures (Fürst, 2016).

Also, commercial imperatives are basically perceptions of how media markets will develop in the future, including bets on future media technologies. One example is media conglomerates buying start-ups and providing huge amounts of venture capital betting on "the next big thing." Silicon Valley is arguably built on venture capital, and most online media companies are still far from profitable. Algorithms might be used to help find promising patterns based on data. This is nothing new, but exactly how, for example, the tourist region of the Mexican "Riviera Maya" around Cancún was identified and developed in the 1970s (Collins, 1979). However, what is perceived as a commercial imperative is largely a result of narrative construction, symbolic 
recomposition, hypothetical resolution, and experimental enactment performed by human actors. One example of the cultural and interpretive impact on what is considered a commercial imperative would be the technological optimism from which "radical new visions of a technological future emerged" that "helped stimulate the creation of privately funded research institutes and investment from high-tech entrepreneurs" in California from the 1970s onward (McCray, 2012, p. 348). Experimental approaches also may have an effect on the practical evaluation of current challenges, which leads to trial-and-error business models. In an uncertain and complex economic situation such as the one we find in hybrid media systems and their economy, decisions are often based on deliberations - direct or indirect exchanges with other actors or business consultants in the industry, an industry that shows significant growth (The Economist, 2013).

\subsection{Technological affordances}

Gibson introduced the concept of affordances in 1977 to discuss how individuals adopted and made use of objects. With reference to animals, he argued that a rock, for example, could be used differently because different animals might perceive different sets of activities for which the rock would be useful. Similarly, Gibson claims that people do not interact with an object without perceiving what it is good for. Hence, affordances are unique to the perceptions of the user, as well as the norms and values governing such perceptions. It thus becomes obvious that affordances are shaped by iterations, i.e. the communicator's and the user's habits, routines, selective attention/exposure patterns, and media repertoires learned and adapted over their life span. This is what Altheide (2014) refers to as "the media spiral": 
Coverage of events at Time 1 will be implicated in how events are organized and carried out and communicated at Time 2 . This is a view of history as reflected not only in mass media coverage, but also the advent of new information technologies that have provided new formats of information, which also serve to promote more social control and surveillance. (p. 11)

With regard to projections, expectations of future media and communication channels also play a role, as Chan-Olmsted, Rim and Zerba (2013) point out: "Beliefs about a new technology tend to determine a person's attitude toward using that technology, which in turn influences his or her intention to use it" (p. 128). For instance, velocity was considered harmful in many ways in the early years of trains and cars (Schivelbusch, 2000), and still today clerics in Saudi Arabia argue that it harmed women's fertility (Jamjoom, 2013). Many experts consider video games with explicit or violent content to be harmful to teenagers and call for a reduction in or regulation of exposure to those games (e.g. Anderson, 2016). Present evaluations also play a role in how humans shape and are shaped by technological affordances: The affordances of social media platforms are a process of negotiation between the technological possibilities of the platform and the decisions of the designers of the services of the platform. Moreover, these designer-influenced technological services are influenced by those who choose to use them. Van Dijck and Poell (2013) point to the central role of the designer, namely triggering user interaction by shaping content on social media platforms: "Now that the flow has taken an 'algorithmic turn,' content is not just programmed by a central agency, even if this agency still has considerable control; users also participate in steering content" (p. 6). Bucher and Helmond (2017) underline that a "user" cannot be reduced to the end-user, but that technological affordances vary for a broader range of user types: "Twitter affords different things to an end-user than to 
a developer, advertiser or researcher. These different user types are predefined by Twitter, by addressing them via distinct interfaces such as the end-user interfaces including the website and Twitter apps, the Twitter APIs for developers and researchers, and the Advertising API for advertisers" (p. 23). It is worth remembering that the Internet was originally designed for military use, but a practical evaluation of how best to use the system, user input, and other complex situations has shaped the Internet and social media into what they are today. In other words, affordances result from a process of interaction between two or more types of human actors (designers in organizations and users) and the technological structure between them. The power relation between the users and the designers is not equal, however. Two different options have emerged: The rich and the have-nots of digital modernity (Schirrmacher, 2015, p. 649), i.e. those behind the screen, who know how to translate their interests and ideals into algorithms, and those in front of the screen, who can only use and adapt to the opportunities that these media offer them. These actors may have very different ideas about what the medium's affordances are. While the user in front of the screen may think a social media platform's affordance is to network and stay connected with friends and family, the designer on the other side may think that a social media platform's affordance is to gather as much personalized and marketable user data as possible. Hence, we tap into the pertinent question of who has power.

\section{Conclusion}

In this article, we have discussed how human agency operates in all dimensions and elements of network media logic. We focused on network media logic and agency, not 
mass media logic - although, with regard to the overlapping of mass media and network media logic one could argue that algorithms also impact mass media logic (as an outcome of network media logic), i.e. by pushing mass media logic towards operating modes involving automation and datafication. How we relate to media is governed by the logics/combination of logics on media platforms in terms of rules and processes of media production, distribution, and usage, as well as our perception of such norms and processes. Human agency is present in all elements of network media logic that cannot be replaced by algorithms, because algorithms are non-neutral. Algorithms are deeply dependent on human actors, especially in the first step; the input/design phase. The calculations themselves and the outcomes they produce are less dependent on human intervention. This has raised questions of who or what can be accountable when algorithmic calculations go wrong, have unintended or undesirable effects. In this article we have argued that algorithms themselves cannot be hold accountable since they arguably have no intentions and lack the reflexive and evaluative element of agency.

Different power configurations are taking shape today - power relations that a purely micro-level analysis and discussion would hide. If we agree that human agency permeates network media logic, we indeed need to direct more attention not only to the users of network media but also to the programmers and designers of the algorithms governing the social media platforms we use, and the institutional contexts and conditions in which they perform the programming. But while algorithms cannot always handle situations as their designers and programmers would have wanted them to, they remain responsible for the algorithms they created. It only shows the limits of programmers' forecasting capabilities in increasingly opaque situations in the Global 
Age (Poveda and Svensson, 2016). Kraemer, van Overveld and Petersen (2011, p. 251) argue that it is reasonable to maintain that software designers are morally responsible for the algorithms they design. But we also need to move up to the meso-level of tech companies and media organizations for a structural perspective, particularly for a debate on algorithmic accountability. This would imply that Google and others cannot hide behind the algorithm when it performs in a racist manner. Google's algorithm is nothing more than Google and the choices the company and its employees make. In this regard, the question as to whether and to what extent algorithms have agency and whether/how we can distinguish between algorithms as actors and algorithms as actions is highly relevant for discussions about media accountability and media regulation, and thus, for Internet governance.

On the macro-level of the public sphere and society, it seems that we have the wrong nightmares about the impact of algorithms on society. Instead of fearing that machines will take over the world (the so-called singularity), we should start to focus on the humans and organizations behind the machines and what structures and conditions shape their actions. And if humans in organizations "control technological development and not vice versa, then technology is neither the hero nor the villain of modernity, but merely one participant in cultural change. Thus, understanding technology in its cultural context is a way to avoid the pitfalls of blind optimism or pessimism about the technological future" (McOmber, 1999, p. 138). It would seem that the whole singularity scare is an in-front-of-the-screen imaginary (to use the wording of Mansell, 2012). When scholars of algorithmic/data journalism paint a picture of news stories being produced without human interference (see, for example, van Dalen, 2012, p. 648), 
they tend to forget about the humans, structures and relations behind the screen. Many authors write entire articles about the governance of algorithms without mentioning their makers, apart from calling on them to design in a more open and democratic way. It becomes rather telling when DeVito (2016) uses an impressive content analysis only to disentangle the values coded into the algorithms selecting news stories for users on Facebook. Indeed, algorithms are not public goods. Programmers and designers are not always available for researchers, and algorithms are often deliberately obscured to protect intellectual property (see DeVito, 2016). Hence, we have to get to the algorithms through the back door, through the results of their programmed steps of computation. In this sense, our argument resonates with Napoli's (2014) call for an understanding of the social construction of algorithms. Our addition to this argument is that it has to start by attending to and unveiling the human actors behind the algorithms, their organizational settings, and what goes into their practices.

Arguments that technology had agency on its own hide the individuals, structures and relations in power and thus serve their interests, interests that become increasingly blurred. To argue that algorithms have agency on their own, agency that is independent of human activity, not only denies the role of media logics but occludes the power inscribed in the algorithm as a structure. Thinking about possible paths for algorithmic accountability points in this direction (Diakopoulos, 2014). This entails keeping in mind both that algorithms are encoded with human intentions and that humans cannot anticipate all the ripple effects of their designs and doings. 


\section{References}

Allen, A (2016) The 'three black teenagers' search shows it is society, not Google, that is racist. The Guardian, 10 June, 16.

Altheide, DL (2014) Media edge: media logic and social reality. New York, NY: Peter Lang.

Ananny, M (2016) Towards an Ethics of Algorithms: Convening, Observation, Probability and Timeliness. Science, Technology, \& Human Values 41(1), pp. 93-117.

Anderson, C (2008) The End of Theory: The Data Deluge Makes the Scientific Method Obsolete. In: Wired. Available at: https://www.wired.com/2008/06/pb-theory/ (accessed 27 April 2018).

Anderson, CW (2013) Towards a sociology of computational and algorithmic journalism. New Media \& Society 15(7), pp. 1005-1021. doi:10.1177/1461444812465137.

Anderson, CA (2016) Media violence effects on children, adolescents and young adults. Health Progress 97(4), pp. 59-62. Available at: https://public.psych.iastate.edu/caa/abstracts/2015-2019/16A2.pdf (accessed 14 April 17).

Arendt, H (1958) The human condition. Available at: http://sduk.us/afterwork/arendt_the_human_condition.pdf (accessed 14 April 17).

Barocas, S, Hood, S and Ziewitz, M (2013) Governing Algorithms: A Provocation Piece. In Governing Algorithms Conference, New York, May 2013.

Bell, E (2016) How Facebook swallowed journalism. Available at: http://en.ejo.ch/short-stories/facebook (accessed 14 April 2017).

Bennett, LW and Segerberg, A (2012) The logic of connective action. Information, Communication and Society 15(5), pp. 739-768. doi:10.1080/1369118X.2012.670661

Big Think Editors (2015) Carl Bernstein: The "Golden Age" of Investigative Journalism Never Existed. Available at: http://bigthink.com/the-voice-of-big-think/carlbernstein-the-golden-age-of-investigative-journalism-never-existed (accessed 14 April 2017).

boyd, d and Crawford, K (2012) Critical questions for big data: provocations for a cultural, technological, and scholarly phenomenon. Information, Communication \& Society 15(5), pp. 662-679. doi:10.1080/1369118X.2012.678878

Bozdag, E (2013) Bias in algorithmic filtering and personalization. Ethics Information Technology 15, pp. 209-227. Berlin: Springer

Bucher, T and Helmond, A (2017) The affordances of social media platforms. In: Burgess, J, Poell, T and Marwick, A (eds) The SAGE Handbook of Social Media. London and New York: SAGE. Available at: http://www.academia.edu/27438172/The_Affordances_of_Social_Media_Platfor ms (accessed 14 April 2017).

Chan-Olmsted, S, Rim, H and Zerba, A (2013) Mobile news adoption among young adults examining the roles of perceptions, news consumption, and media usage. Journalism \& Mass Communication Quarterly 90(1), pp.126-147. doi:10.1177/1077699012468742. 
Collins, CO (1979) Site and situation strategy in tourism planning: A Mexican case study. Annals of Tourism Research 6(3), pp. 351-366. doi:10.1016/01607383(79)90109-9.

DeVito, MA (2016) From editors to algorithms. Digital Journalism 5(6), pp. 753-773. doi:10.1080/21670811.2016.1178592.

Diakopoulos, N (2014) Algorithmic accountability reporting: On the investigation of black boxes. Tow Center for Digital Journalism, Columbia University.

Diakopoulos, N (2016) Accountability in Algorithmic Decision Making. Communications of the ACM 59 (2).

Emirbayer, M and Mische, A (1998) What is agency? American Journal of Sociology, 103(4), pp. 962-1023. https://doi.org/10.1086/231294.

Esser, F (2013) Mediatization as a challenge: media logic versus political logic. In: Kriesi, H, Lavenex, S, Esser, F, Matthes, J, Bühlmann, M and Bochsler, D (eds) Democracy in the Age of Globalization and Mediatization. Basingstoke: Palgrave-Macmillan, pp. 155-176.

Gibson, JJ (1977) The theory of affordances. In: Shaw, R and Bransford, J (eds) Perceiving, acting, and knowing: Toward an ecological psychology. Hillsdale, NJ: Erlbaum, pp. 67-82.

Gillespie, T (2016) Algorithms, clickworkers, and the befuddled fury around Facebook Trends. Available at: http://culturedigitally.org/2016/05/facebooktrends/\#sthash.Sp9Qj1Xi.dpuf (accessed 14 April 2017).

Gillespie, T and Seaver, N (2016) Critical algorithm studies: a reading list. Available at: https://socialmediacollective.org/reading-lists/critical-algorithm-studies/ (accessed 14 April 2017).

Gillespie, T (2014) The relevance of algorithms. In: Gillespie, T, Boczkowski, PJ and Foot, KA (eds) Media Technologies: Essays on Communication, Materiality, and Society. Cambridge. MA: MIT Press, pp. 167-194.

Goodwin, DK (2014) The bully pulpit: Theodore Roosevelt, William Howard Taft, and the golden age of journalism. New York: Simon and Schuster.

Gurevich, Y (2012) What is an algorithm? In: SOFSEM'12 Proceedings of the 38th international conference on Current Trends in Theory and Practice of Computer Science, pp. 31-42.

Habermas, J (1968) Technik und Wissenschaft als "Ideologie"? Man and World 1(4), pp. 483-523. doi:10.1007/BF01247043

Helft, M (2011) Facebook, foe of anonymity, is forced to explain a secret. The New York Times, 13 May, $11 . \quad$ Available at: http://www.nytimes.com/2011/05/14/technology/14facebook.html?_r=1 (accessed 14 April 17).

Hindi, R (2017). How my research put my dad out of a job. In: Medium. Available at: https://medium.com/snips-ai/how-my-research-in-ai-put-my-dad-out-of-a-job1a4c80ede1b0 (accessed 27 April 2018)

Hjarvard, S (2013) The Mediatization of Culture and Society. London: Routledge.

Huffington Post (2010) Google CEO On Privacy (VIDEO): 'If You Have Something You Don't Want Anyone To Know, Maybe You Shouldn't Be Doing It'. Available at: $\quad$ http://www.huffingtonpost.com/2009/12/07/google-ceo-on-privacyif_n_383105.html_(accessed 14 April 17).

Jamjoom, $\bar{M}$ (2013) Saudi cleric warns driving could damage women's ovaries. CNN Online, $13 \quad$ September

13. Available at: 
http://edition.cnn.com/2013/09/29/world/meast/saudi-arabia-women-drivingcleric/ (accessed 14 April 17).

Jansen, T (2016) Who Is Talking? Some Remarks on Nonhuman Agency in Communication. Communication Theory 26, pp. 255-272. doi:10.1111/comt.12095.

Jungherr, A, Schoen, H, Posegga, O and Jürgens, P (2016) Digital trace data in the study of public opinion. An indicator of attention toward politics rather than political support. Social Science Computer Review. doi:10.1177/0894439316631043.

Just, N and Latzer, M (2016) Governance by algorithms: reality construction by algorithmic selection on the Internet. Media Culture \& Society. doi:10.1177/0163443716643157.

Kay, M, Matuszek, C, and Munson, SA (2015) Unequal representation and gender stereotypes in image search results for occupations. In: Proceedings of the 33rd Annual ACM Conference on Human Factors in Computing Systems. ACM, 3819-3828.

Kitchin, R. (2017). Thinking critically about and researching algorithms. Information, Communication \& Society 20(1), pp. 14-29.

Kraemer, F, van Overveld, K and Petersen M (2011) Is there an ethics of algorithms? Ethics and information technology 13(3), pp. 251-260. doi:10.1007/s10676-0109233-7.

Kowalski, R (1979) Algorithm = logic + control. Communications of the ACM. 22(7), pp. 425- 436. doi:10.1145/359131.359136.

Langlois, G, and Elmer, G (2013) The Research Politics of Social Media Platforms. Culture Machine, 14.

Latour, B (1996) On actor-network theory: a few clarifications. Soziale Welt, pp. 369381. Available at: http://www.jstor.org/stable/40878163 (accessed 14 April 17).

Lewis, SC and Westlund, O (2015) Actors, actants, audiences, and activities in crossmedia news work. Digital Journalism 3(1), pp. 19-37. doi:10.1080/21670811.2014.927986.

Mansell, R (2012) Imaginging the Internet. Communication innovation and governance. Oxford: Oxford University Press.

Marcuse, H (1965) Industrialization and capitalism. New Left Review 30, pp. 3-18.

McCray, P (2012) California dreamin': Visioneering the technological future. In Janssen, V (ed.) Minds and Matters: Technology in California and the West. Huntington: University of California Press, pp. 347-378.

McOmber, J B (1999) Technological autonomy and three definitions of technology. Journal of Communication, 49(3), pp. 137-153. https://doi.org/10.1111/j.14602466.1999.tb02809.x

Mitcham, C (2014) Agency in Humans and in Artifacts: A contested discourse. In: Kroes, P and Verbeek, PP (eds) The Moral Status of Technical Artefacts. Philosophy of Engineering and Technology 17. Berlin: Springer, pp. 11-29.

Nahon, K and Hemsley, J (2013) Going Viral. Cambridge: Polity Press.

Napoli, PM (2014) Automated media: An institutional theory perspective on algorithmic media production and consumption. Communication Theory 24(3): pp. 340-360.

Neff, G and Nagy, P (2016). Talking to Bots. Symbiotic Agency and the case of Tay. International Journal of Communication 10(2016), pp. 4915-4931. 
Poveda, O and Svensson, J (2016). Re-thinking the Global Age as Interdependence, Opacity and Inertia. Triple C 14(2), pp. 475-495.

Sandvig, C, Hamilton, K, Karahalios, K and Langbord, C (2016) When the Algorithm itself Is a Racist. Diagnosing Ethical Harm in the Basic Components of Software. International Journal of Communication 10(2016), pp. 4972-4990.

Schirrmacher, F (2015) Das Armband der Nellie Kroes. In: Schirrmacher, F (ed) Technologischer Totalitarismus. Eine Debatte. Berlin: Suhrkamp, pp. 62-69.

Schivelbusch, W (2000) Geschichte der Eisenbahnreise. Zur Industrialisierung von Raum und Zeit im 19. Jahrhundert. Frankfurt: Fischer.

Seaver, N (2013) Knowing algorithms. Media in Transition 8, Cambridge, MA, Available at: http://nickseaver.net/papers/seaverMiT8.pdf (accessed 14 April 2017).

Steiner, C (2012) Automate this. How algorithms came to rule our world. New York: Penguin Books.

Striphas, T (2015) Algorithmic Culture. European Journal of Cultural Studies 18(4-5), pp. 395-412.

Strömbäck, J and Esser, F (2014) Mediatization of politics: Transforming democracies and reshaping politics. In: Lundby, K (ed) Mediatization of Communication. Berlin: De Gruyter Mouton, pp. 374-404.

The Economist (2013) To the brainy, the spoils. As the world grows more confusing, demand for clever consultants is booming. 11 May, 17. Available at: http://www.economist.com/news/business/21577376-world-grows-moreconfusing-demand-clever-consultants-booming-brainy (accessed 14 April 2017).

Thielman, S (2016) Facebook fires trending team, and algorithm without humans goes crazy. The Guardian, 29 August, 16). Available at: https://www.theguardian.com/technology/2016/aug/29/facebook-fires-trendingtopics-team-algorithm?CMP=fb_gu (accessed 14 April 2017).

Tufekci, Z (2015) Algorithmic harms beyond Facebook and Google: Emergent challenges of computational agency. Journal on Telecommunication \& High Technology Law 13, pp. 203 -218.

Van Dalen, A (2012) The Algorithms behind the headlines. Journalism Practice 6 (5-6), pp. 648-658. doi:10.1080/17512786.2012.667268.

Van Dijck, J (2013) The Culture of Connectivity: A Critical History of Social Media, Oxford: Oxford University Press.

Van Dijck, J and Poell, T (2013) Understanding social media logic. Media and Communication 1(1), pp. 2-14. doi: 10.17645/mac.v1i1.70. 
The End of Media Logics? On Algorithms and Agency

Figure 1 Media Logic: Dimensions and Agency 
The End of Media Logics? On Algorithms and Agency

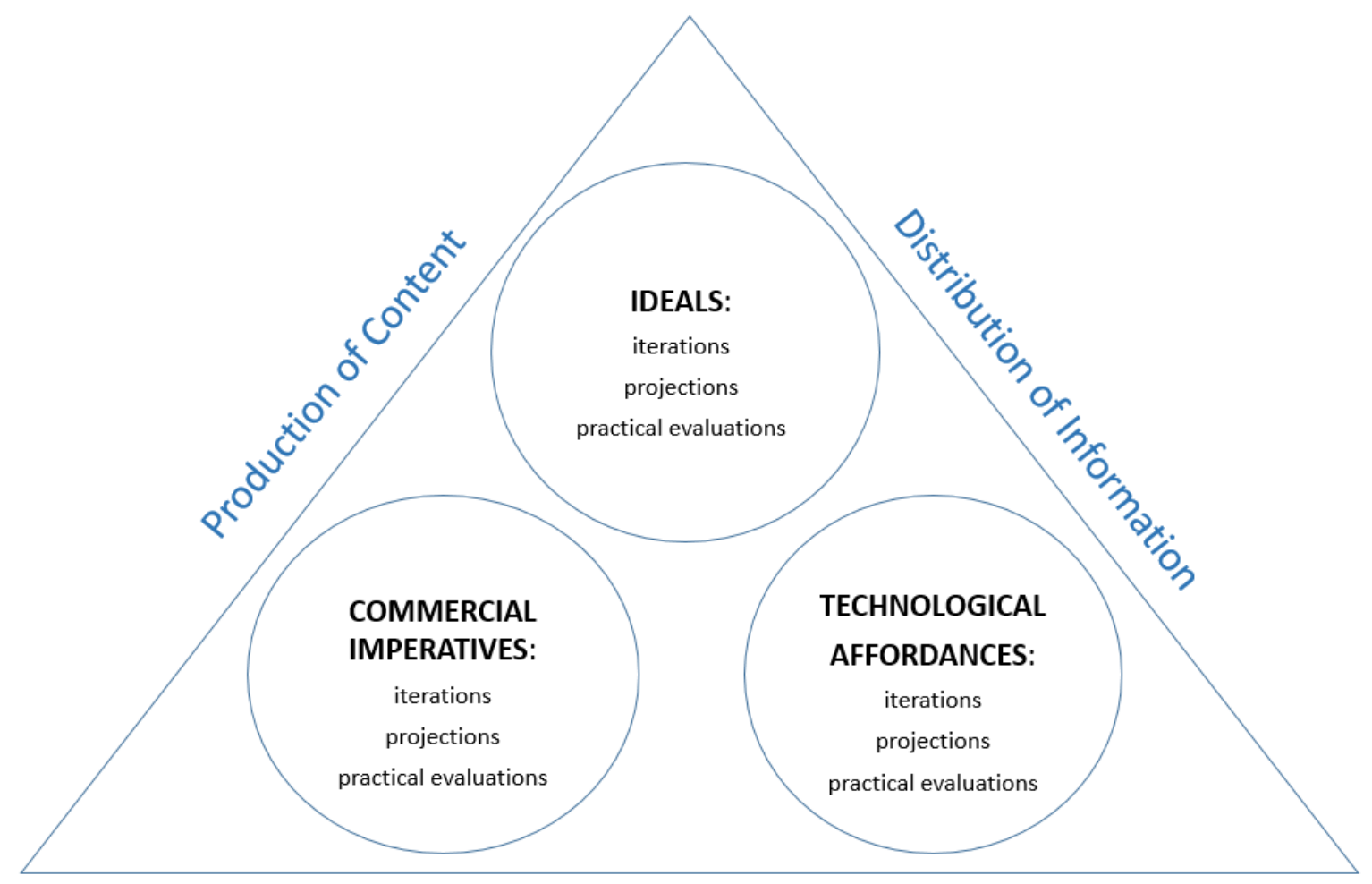

Media Use 